\title{
COMPARISION OF LIFITING RE-ENTRY AND BALLISITC RE-ENTRY
}

\section{A. RATHAN BABU ${ }^{1}$, P. VIJAY KUMAR ${ }^{2}$, B. PRAVEEN ${ }^{3}$ \& R. SURESH KUMAR ${ }^{4}$}

${ }^{1,4}$ Assistant Professor, Department of Aeronautical Engineering, Institute of Aeronautical

Engineering, Hyderabad, Telangana, India

${ }^{2}$ Department of Aeronautical Engineering, Institute of Aeronautical Engineering, Hyderabad, Telangana, India

${ }^{3}$ Assistant Professor, Department of Aeronautical Engineering, MLR Institute of Technology, Hyderabad, Telangana, India

\section{ABSTRACT}

This paper highlights comparison of lifting re-entry and ballistic re-entry. 3-D. $O$. F code is developed and used for comparing the different re-entries. The main objective of this paper is to minimize deceleration on re-entry body and find the optimal flight path angle ranges to escape under and overshoot conditions of re-entry.

KEYWORDS: Lifting Re-entry, Ballistic Re-entry, Flight Path Angle \& Drag Coefficient

Received: Mar 12, 2018; Accepted: Apr 02, 2018; Published: Apr 26, 2018; Paper Id.: IJMPERDJUN201812

\section{INTRODUCTION}

Re-entry is used for different purposes like planetary and interplanetary, and re-entry is categorized into three types i.e., lifting, ballistic, skip re-entry. During lifting re-entry lift coefficient is not zero and it is assumed to be a small value. In re-entry, the major aerodynamic force is a drag, which plays a major role in the decelerating body from high speeds in the order of $8-10 \mathrm{~km} / \mathrm{s}$ to terminal velocity. During ballistic re-entry lift coefficient is zero and body fall on the surface of the planet under the influence of gravitational force. In skip re-entry, sometimes body lifts off and returns to its state. In this process, body possess lift at some instant such that P. E is increased and K. E is decreased for the conservation of total energy.

During re-entry, body has a maximum deceleration which depends on initial flight path angle and entry velocity. If flight path angle is increased then body undergoes the steepest re-entry. For manned mission, re-entry deceleration level shouldn't increase beyond '4g' level. When a body is entering the planet's atmosphere where density level is increased such that drag on the body will increase. Therefore, deceleration level is increased and heat load on the body will also increase. Deceleration level on the body is proportional to the square of the velocity and heat load on the body is proportional to the cube of velocity. Flow classification on the body is based on the Knudsen number( $\mathrm{Kn})[4] . \mathrm{Kn} \geq 10$ is considered as (free-molecular), whereas $0.01 \leq \mathrm{Kn} \leq 10$ is considered (transition), and $\mathrm{Kn}<0.01[2]$ is considered as(continuum-flow)

$$
. \mathbf{K n}=\frac{\lambda}{\mathbf{L}}
$$




\section{METHODOLOGY}

The equations of motion [1], [4] for the atmospheric phase are standard and have been developed in a non-inertial frame of the planet. The kinematic equations of motion of point mass are given by:

$$
\begin{aligned}
& \dot{\boldsymbol{\delta}}=\frac{\mathrm{V} \cos \gamma \cos \chi}{\mathrm{R} \cos \lambda} \\
& \dot{\lambda}=\frac{\mathrm{V} \cos \gamma \sin \chi}{\mathrm{R}} \\
& \dot{\mathbf{R}}=\mathrm{V} \sin \gamma
\end{aligned}
$$

The kinetic equations of motion of point mass is given by

$$
\begin{aligned}
& \dot{\mathrm{V}}=-\mathrm{g} \sin \gamma+\frac{\mathrm{F}_{\mathrm{Y}}}{\mathrm{m}}+\Omega^{2} \mathrm{R} \cos \lambda(\sin \gamma \cos \lambda-\cos \gamma \sin \chi \sin \lambda) \\
& \dot{\gamma}=\left(\frac{\mathrm{V}}{\mathrm{R}}-\frac{\mathrm{g}}{\mathrm{V}}\right) \cos \gamma+\frac{\mathrm{F}_{\mathrm{X}}}{\mathrm{Vm}}+2 \Omega \cos \chi \cos \lambda+\frac{\Omega^{2} \mathrm{R} \cos \lambda(\cos \gamma \cos \lambda+\sin \gamma \sin \chi \sin \lambda)}{\mathrm{V}} \\
& \dot{\chi}=\frac{-\mathrm{V} \cos \gamma \cos \chi \tan \lambda}{\mathrm{R}}+\frac{\mathrm{F}_{\mathrm{Z}}}{\mathrm{mV} \cos \gamma}+2 \Omega(\tan \gamma \sin \chi \cos \lambda-\sin \lambda)-\frac{\Omega^{2} \cos \chi \cos \lambda \sin \lambda}{\mathrm{V} \cos \gamma}
\end{aligned}
$$

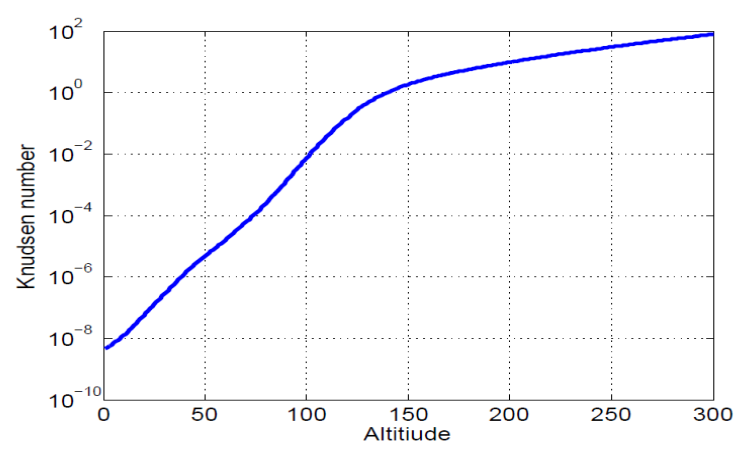

Figure 1: Altitude Vs Knudsen Number for Characteristic Length of $1.7 \mathrm{~m}$

\section{RESULTS AND DISCUSSIONS}

For validation of code developed, simulations are started from an altitude of $300000 \mathrm{~m}$ with a flight path angle $11.72^{0}$ and initial velocity $12000 \mathrm{~m} / \mathrm{s}$. Figure 2 shows the comparison of present trajectory simulation results with trajectory data with ref [1], [5]. For re-entry, the mass of a body is assumed to be $1000 \mathrm{~kg}$, with an entry velocity of $8000 \mathrm{~m} / \mathrm{s}$ at an altitude $100000 \mathrm{~m}$. Flight path angle ranges from $-1 \mathrm{deg}$ to $-6 \mathrm{deg}$. An upper limit of $-6 \mathrm{deg}$ is chosen because the deceleration of body depends on flight path angle and entry velocity. If flight path is beyond -6deg the deceleration and heat load on the body is too high therefore it is considered to be the upper limit. From -0.1deg it is feasible for a body to have safe re-entry. In this paper, we just took from -1deg so that time for re-entry will be less and it is seen that deceleration and heat loads are in the limit. If we take positive value of flight path angle i.e., 1 deg the body undershoots. If we increase flight path angle then body overshoots, so from trials we have fixed the limits of flight path angle from - 0.1 to $-6 \mathrm{deg}$.

Atmospheric model is assumed to be exponential [3] and isothermal temperature conditions. Figure 3 \& 4 shows the difference between lifting and ballistic re-entry in which lifting re-entry has some glide time such that time taken for reentry of the body is more compared to ballistic. From figures 5,6,7,8 it is observed that lifting re-entry has deceleration 
level is less compared to ballistic. Figure 9 shows the heat flux which are in limits and it is less for a lifting re-entry. It is observed that during re-entry first there is an increase in deceleration due to increase in density and it goes to the maximum at an altitude then it decreases because of a decrease in velocity of the body.

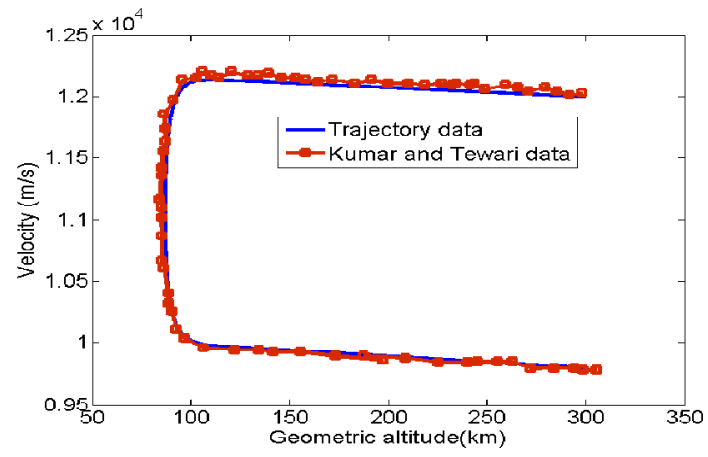

Figure 2: Validation of 3D. O. F Code

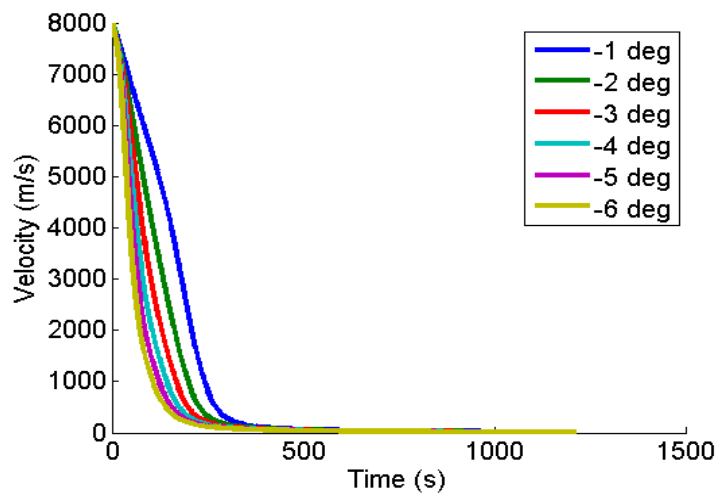

Figure 3: Velocity Vs Time for a Lifting Re-entry

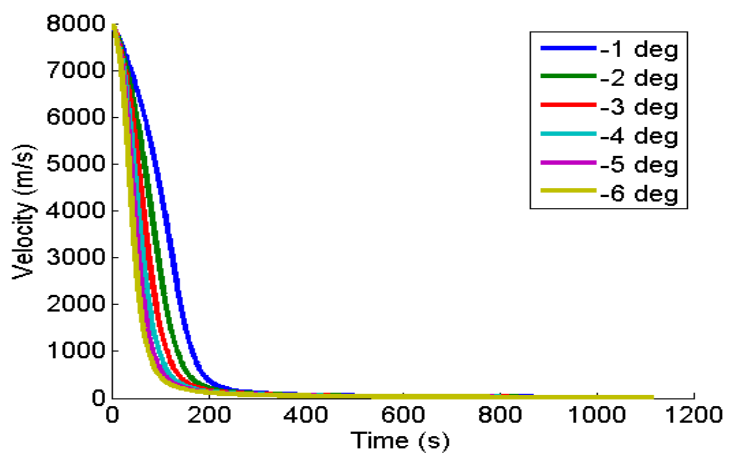

Figure 4: Velocity Vs Time for a Ballistic Re-entry 


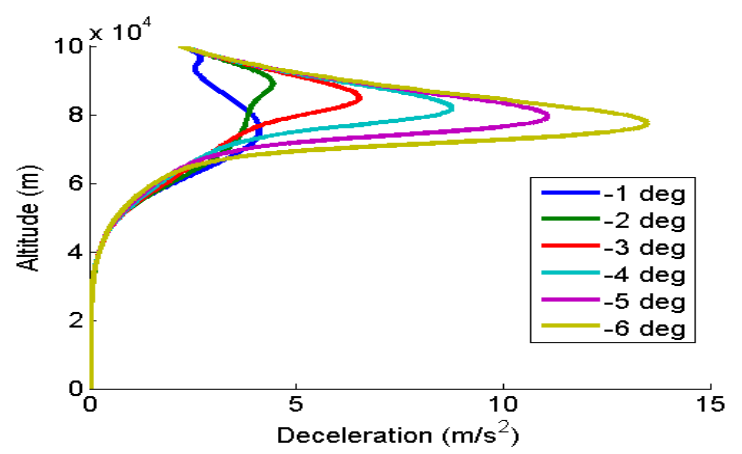

Figure 5: Deceleration Vs Altitude for a Lifting Re-entry

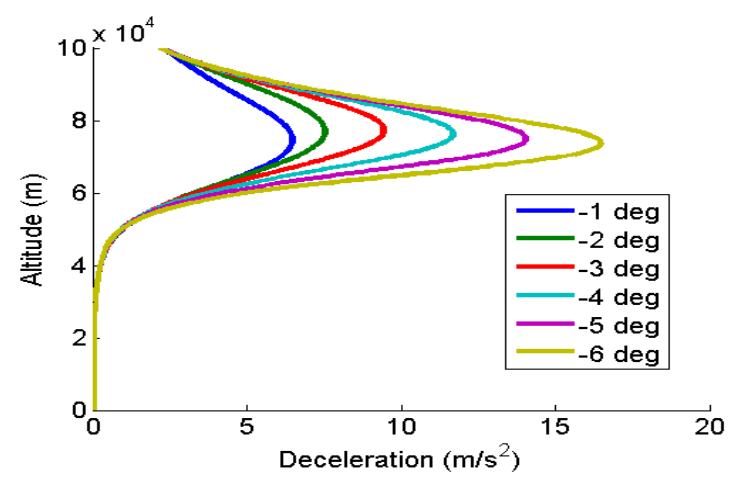

Figure 6: Deceleration Vs Altitude for a Ballistic Re-entry

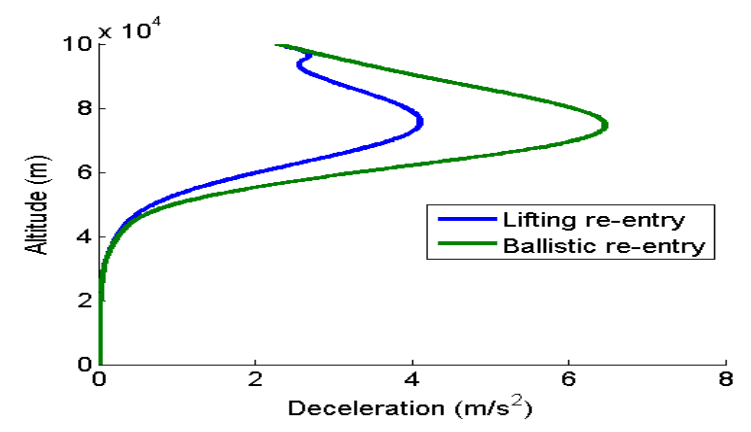

Figure 7: Comparison of Deceleration Vs Altitude for a Lifting and Ballistic Re-entry at -1 Deg Flight Path Angle

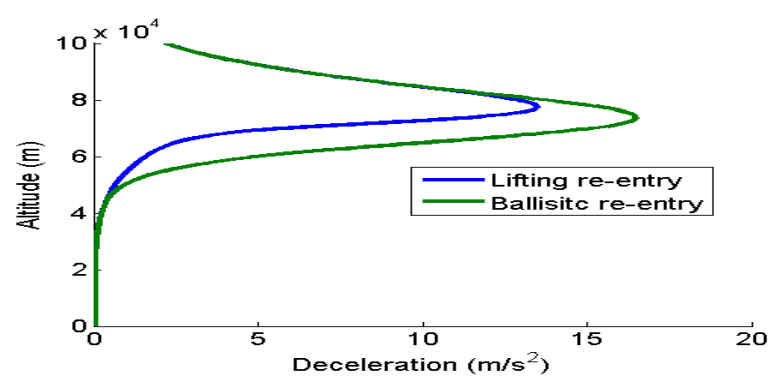

Figure 8: Comparison of Deceleration Vs Altitude for a Lifting and Ballistic Re-entry at -6 Deg Flight Path Angle

\section{CONCLUSIONS}

It observed that for re-entry the deceleration level and heat load depends on entry velocity and initial flight path angle and it is independent of the mass of the body. From trajectory simulations, it is seen that from -0.1 to $-6 \mathrm{deg}$ of flight 
path angles are optimal for both ballistic and lifting re-entry which are obtained from serval trials of trajectory run. For the body to have less deceleration level and more touch down time lifting re-entry is preferred than ballistic.

\section{REFERENCES}

1. Mrinal Kumar and Ashish Tewari. Trajectory and attitude simulation for aerocaptureand aerobraking. Journal of spacecraft and rockets, 42(4):684-693, 2005.

2. RathanBabu, U. Shiva Prasad, CH. Satya Sandeep, R. Suresh Kumar, Aerodynamics and Stability of Spacecraft during Earth entry. International Journal of Mechanical Engineering and Technology, 8(6), 2017, pp. 796-803.

3. G. E. Cook.,"The Aerodynamic Drag of Near Earth Satellites", AERONAUTICAL RESEARCH COUNCIL, MINISTRY OF AVIATION,1960.

4. John David Anderson. Hypersonic and high temperature gas dynamics. Aiaa, 2000.

5. Frank J Regan. Dynamics of atmospheric re-entry. Aiaa, 1993.

6. Mrinal Kumar and Ashish Tewari. Trajectory and attitude simulation for mars aerocaptureand aerobraking. Journal of spacecraft and rockets, 43(3):585-593, 2006. 
